\title{
Carbon Nanotubes as Thermally Induced Water Pumps
}

Oyarzua, Elton; Walther, Jens Honore; Megaridis, Constantine M; Koumoutsakos, Petros; Zambrano, Harvey A

Published in:
A C S Nano

Link to article, DOI:

10.1021/acsnano.7b04177

Publication date:

2017

Document Version

Peer reviewed version

Link back to DTU Orbit

Citation (APA):

Oyarzua, E., Walther, J. H., Megaridis, C. M., Koumoutsakos, P., \& Zambrano, H. A. (2017). Carbon Nanotubes as Thermally Induced Water Pumps. A C S Nano, 11(10), 9997-10002.

https://doi.org/10.1021/acsnano.7b04177

\section{General rights}

Copyright and moral rights for the publications made accessible in the public portal are retained by the authors and/or other copyright owners and it is a condition of accessing publications that users recognise and abide by the legal requirements associated with these rights.

- Users may download and print one copy of any publication from the public portal for the purpose of private study or research.

- You may not further distribute the material or use it for any profit-making activity or commercial gain

- You may freely distribute the URL identifying the publication in the public portal

If you believe that this document breaches copyright please contact us providing details, and we will remove access to the work immediately and investigate your claim 


\title{
Carbon Nanotubes as Thermally-Induced Water Pumps
}

Elton Oyarzua, ${ }^{\dagger}$ Jens Honore Walther, ${ }^{\ddagger} \uparrow$ Constantine M Megaridis, ${ }^{\S}$ Petros Koumoutsakos, ${ }^{\top}$ and Harvey A. Zambrano*,†

$\dagger$ Department of Chemical Engineering, Universidad de Concepcion, Concepcion, Chile $\ddagger$ Department of Mechanical Engineering, Technical University of Denmark, DK-2800 Kgs. Lyngby, Denmark

IComputational Science and Engineering Laboratory, Department of Mechanical and Process Engineering, ETH Zurich, CH-8092 Zurich, Switzerland $\S$ Department of Mechanical and Industrial Engineering, University of Illinois at Chicago,

E-mail: harveyzambrano@udec.cl

Phone: +56 (0)41 2201468

\author{
Chicago IL, USA
}

Phone:

\begin{abstract}
Thermal Brownian Motors (TBMs) are nanoscale machines that exploit thermal fluctuations to provide useful work. We introduce a TBM-based nanopump which enables continuous water flow through a Carbon Nanotube (CNT) by imposing an axial thermal gradient along its surface. We impose spatial asymmetry along the CNT by immobilizing certain points on its surface. We study the performance of this molecular motor using Molecular Dynamics (MD) Simulations. From the MD trajectories, we compute the net water flow and the induced velocity profiles for various
\end{abstract}


imposed thermal gradients. We find that spatial asymmetry modifies the vibrational modes of the CNT induced by the thermal gradient, resulting in a net water flow against the thermal gradient. Moreover, the kinetic energy associated with the thermal oscillations rectifies the Brownian motion of the water molecules, driving the flow in a preferred direction. For imposed thermal gradients of $0.5-3.3 \mathrm{~K} / \mathrm{nm}$, we observe continuous net flow with average velocities up to $5 \mathrm{~m} / \mathrm{s}$ inside CNTs with diameters of $0.94,1.4$ and $2.0 \mathrm{~nm}$. The results indicate that the CNT-based asymmetric thermal motor can provide a controllable and robust system for delivery of continuous water flow with potential applications in integrated nanofluidic devices.

\section{Keywords}

Thermal pump, thermal vibrations, single-walled carbon nanotubes, nanofluidics, molecular dynamics.

\section{Keywords}

thermal pump, thermal vibrations, single-walled carbon nanotubes, nanofluidics, molecular dynamics

Recent developments in nanotechnology are enabling the fabrication of devices such as nano Lab-On-a-Chip (LOC) units. ${ }^{1,2}$ These integrated systems hold the promise of combining in a single nanochip and with molecular level resolution, the complete sequence of all technical stages found in traditional clinical laboratories. Nanochannels are an essential part of such systems, as conduits are needed to integrate the functional network components. The development of nanoscale LOC (nLOC) units relies on the rational design of nanochannels conducting the fluids and require a pumping mechanism for driving the flows. Flows in 
nanoconfinement are known to behave differently than flows at the macro- and micro-scale due to dramatic increases of the surface-to-volume ratio. It has been reported that water transport through carbon nanotubes is one to five orders of magnitude faster than predicted by continuum models. ${ }^{3-5}$ In addition to inducing fast water flow, CNTs possess extraordinary mechanical, electronic, thermal and chemical properties, ${ }^{6}$ making them attractive candidates as conduits of nanofluidic devices. At the same time, the mechanisms required to drive water flow in nanoconfined geometries remain the subject of intense research. ${ }^{7}$ It has been reported that large pressure gradients are required to induce flow in CNT based nanomembranes, ${ }^{5,8}$ while electrokinetic flows rely on single-file transport of water molecules in a $\mathrm{CNT}^{9-11}$ and capillarity is not a means to deliver continuous flow. Finally, due to the ultra smoothness of the CNT walls ${ }^{12}$ and their high thermal conductivity, ${ }^{13}$ mass transport inside CNTs (as driven by imposed thermal gradients) has received considerable attention over the past decade. ${ }^{14-25}$

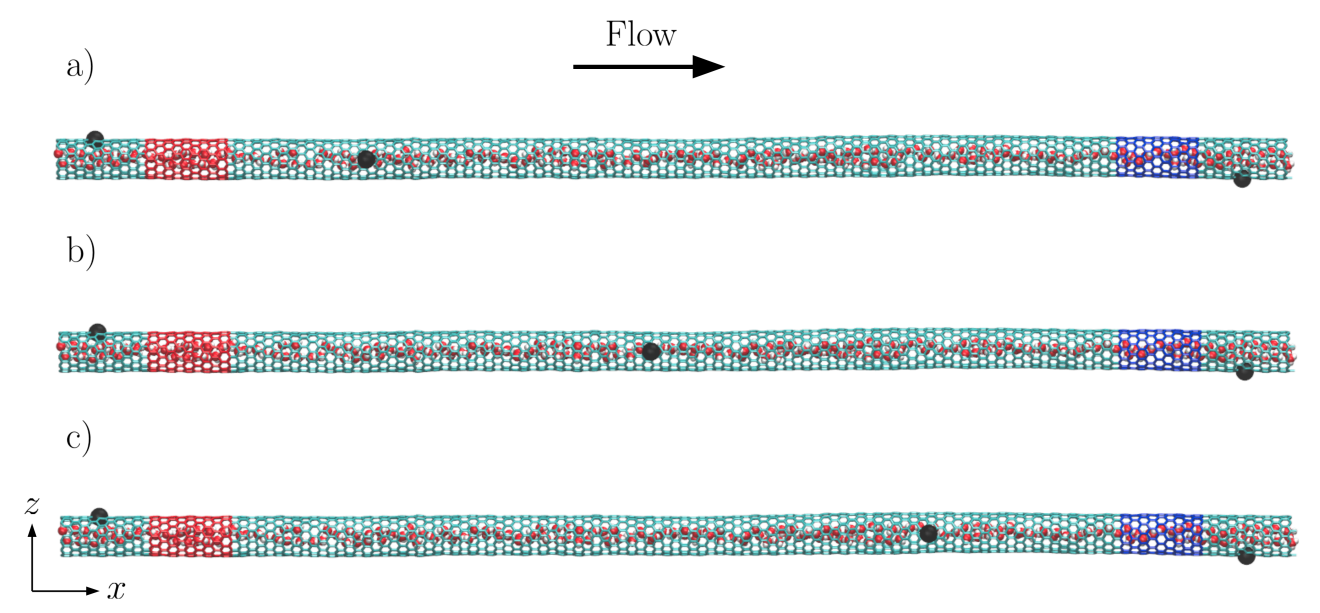

Figure 1: Illustration of the different CNT configurations studied in this work. The black spots indicate the fixed points along each CNT. The red and blue zones represent the heated sections of the CNT. The lengths of the CNTs were either $30 \mathrm{~nm}$ or $60 \mathrm{~nm}$.

(1)
In this work, molecular dynamics (MD) simulations are employed to investigate the continuous flow of water inside a CNT, as driven by an imposed constant thermal gradient. We propose a nanomotor based on the thermal Brownian ratchet concept ${ }^{26}$ to enable fast 
and continuous water flow through a nanoconduit. The device consists of a single-wall CNT filled with water. The CNT is fixed at three points, as shown in Figure 1, with the central fixed point working as a pivot. Two heating zones near the ends impose a thermal gradient along the CNT. The spatial symmetry of the system is broken by the specific position of the fixed points, as depicted in Figure 1. The thermal excitation of the carbon atoms induces oscillations along the CNT with peak amplitudes directly associated to the local temperature. We find that differences in oscillation amplitudes between the higher and lower temperature zones lead to a net water flow opposite to the thermal gradient (along declining temperature). Using this configuration, we systematically investigate the flow dependence on the magnitude of the imposed thermal gradient and the influence of the position of the central fixed point, which breaks the symmetry of the system.

\section{Results and discussion}

We study first a reference case, which consists of a CNT with fixed points at its two ends and its geometric center, as depicted in Figure 1b. The fixed carbon in the middle restricts the position of the CNT without significantly altering the temperature profile along the CNT (Supporting Information Fig. S3). Here, we use a $30 \mathrm{~nm}$ long zig-zag (12,0) CNT, completely filled with water. The CNT is subjected to axial thermal gradients of either 1.6, 2.3 or $3.3 \mathrm{~K} / \mathrm{nm}$. Upon imposing a thermal gradient, we observe the water molecules inside the CNT flow toward the low-temperature zone. Furthermore, by systematically increasing the imposed thermal gradient, we note that the water axial velocity (likewise flow rate) increase linearly, as shown in Figure 2a, with a rate following

$$
v=-1.49 \nabla T
$$

which is consistent with prior studies of thermophoresis in CNTs. ${ }^{15-17}$

The computed net water flow in the CNT is attributed to the thermal oscillations induced 

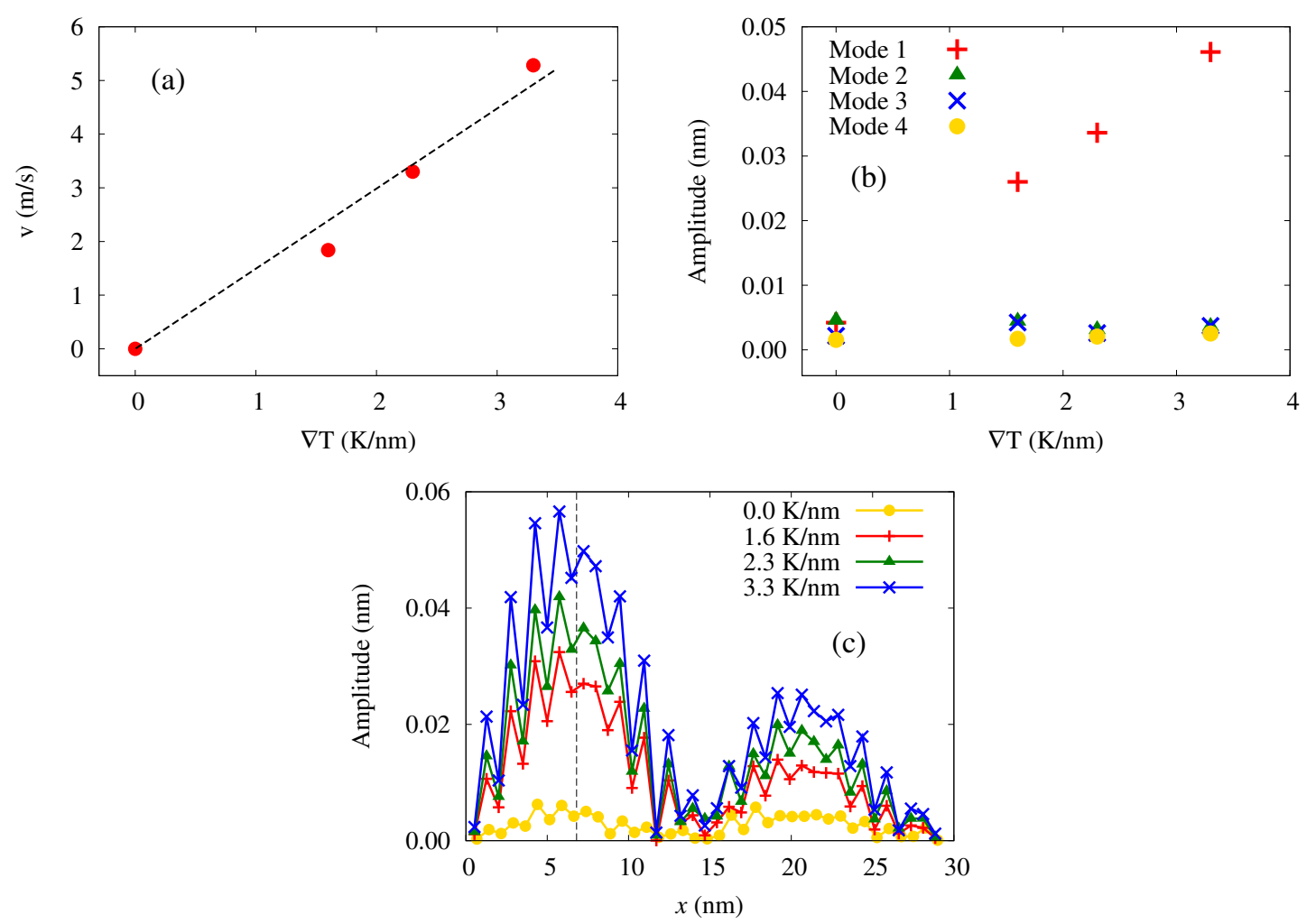

Figure 2: Mean velocities and FFT analysis for the $30 \mathrm{~nm}$-long $(12,0)$ CNT with fixed points as shown in Figure 1b. (a) Mean flow velocities of water for imposed thermal gradients of $0,1.6,2.3$ and $3.3 \mathrm{~K} / \mathrm{nm}$. The dashed line corresponds to a linear fit of the data, under the condition $\mathrm{v}_{(\nabla T=0)}=0 \mathrm{~m} / \mathrm{s}$. (b) Amplitudes of the vibrational modes 1-4 computed at $7 \mathrm{~nm}$ from the left end of the CNT. This position is depicted by the vertical dashed line in Figure 2c. (c) Amplitudes measured for the first mode at three different imposed thermal gradients along the axial direction of the CNT. The zero thermal gradient case $(330 \mathrm{~K})$ is also shown in this figure. 
in the CNT according to the imposed thermal gradient. We find that the particular position of the central fixed point is key to rectify the water motion in a preferential direction, which results in a constant net flow of water. In order to quantify the oscillations of the system, we perform a fast Fourier transform (FFT) analysis to determine the amplitudes and frequencies of the thermally-induced vibrational modes of the CNTs. In particular, for the system illustrated in Figure 1b, we perform the FFT analysis at a point located between the left fixed point and the center point of the CNT, i.e. $7 \mathrm{~nm}$ from the left periodic border, at different imposed thermal gradients. The amplitude values of the first four vibrational modes as functions of the imposed thermal gradients are shown in Figure $2 \mathrm{~b}$. We note that the frequencies of the vibrational modes are associated directly with the size of the system, displaying no relation to the imposed thermal gradients. For example, for the $30 \mathrm{~nm}$-long CNT filled with water, the first four vibrational modes have frequencies of 0.0925 , $0.2100,0.3750$ and $0.4825 \mathrm{THz}$ respectively. Figure $2 \mathrm{~b}$ shows that an increase in the imposed thermal gradient results in larger amplitudes in the vibrational mode 1 . For modes 2, 3 and 4 no change is observed when different thermal gradients are imposed. Therefore, as a thermal gradient is imposed, the induced flow rates depend on the amplitude of the thermal oscillations exclusively in vibrational mode 1 . Indeed, the amplitudes in vibrational mode 1 along the CNT for different imposed thermal gradients are shown in Figure 2c, which shows that the high-temperature zone (left) acquires larger oscillations compared to the lowtemperature zone (right) for all imposed thermal gradients. Specifically, our results indicate that the water flow in the CNT is induced by a continuous whip-like effect generated by the difference in the oscillations of the CNT in the two heated zones. Moreover, as the imposed thermal gradient is increased, the amplitude of the oscillations increases, inducing higher flow rates.

Previous studies ${ }^{27,28}$ have shown that a net flow can be induced inside a CNT imposing traveling waves. Hence, Insepov et al. ${ }^{27}$ imposed Rayleigh traveling waves in a single-walled CNT to transport gas. They observed a time-dependent flow rate with time decay. Likewise, 
Qiu et al. ${ }^{28}$ noted that by applying a periodic force in a cantilever CNT, a water net flow was produced. Moreover, at higher applied forces, greater amplitudes at the free end of the CNT were observed, leading to higher water flow rates. In terms of performance, our TBM converts thermal energy directly into water flow with an efficiency of $c a .0 .2 \%$, similar to the nanopump proposed by Qiu et al. ${ }^{28}$ or the nanomotor studied by Hou et al. ${ }^{19}$ In general, the TBM presented here works with similar efficiency as previously-proposed Brownian motors. ${ }^{29-31}$ The calculation details of the efficiency associated with our TBM/CNT pump are described in the Supporting Information. Furthermore, we propose that the mechanism reported in the present study corresponds to a thermally-rectified motion, as previously observed by Becton and Wang, ${ }^{32}$ who showed that a graphene nanoribbon mounted on a thermalized graphene sheet moved toward the low-temperature zone of the sheet. We infer that the mechanism driving the ribbon on the graphene sheet was the thermally-induced oscillations on the graphene sheet generated by the imposed temperature gradient.

In order to gain insight into the mechanism driving the water flow in the CNT and investigate further the role of the fixed central point, we vary systematically its position as shown in Figure 1. We also conduct simulations for $60 \mathrm{~nm}$-long $(12,0)$ CNTs filled with water under an imposed thermal gradient of $2 \mathrm{~K} / \mathrm{nm}$ and positions of the fixed middle point as shown in Figure 1. Further details of the distances and dimensions used in each simulation are provided in the Supporting Information. For the three different cases, velocity profiles with radial position are shown in Figure 3a. The position of the fixed middle point only slightly modifies the water flow rate. For example, for case (a) (configuration shown in Figure 1a), a lower flow rate is observed. This confirms that the axial flow rate is not exclusively thermal-gradient dependent; there is also strong dependence on the vibrational behavior in the CNT. In order to quantify the vibrational modes for the different cases, a FFT analysis was performed.

The amplitudes measured for the three cases and the vibrational modes in the CNT are shown in Figure 3b, c and d. These figures show that the position of the middle point 

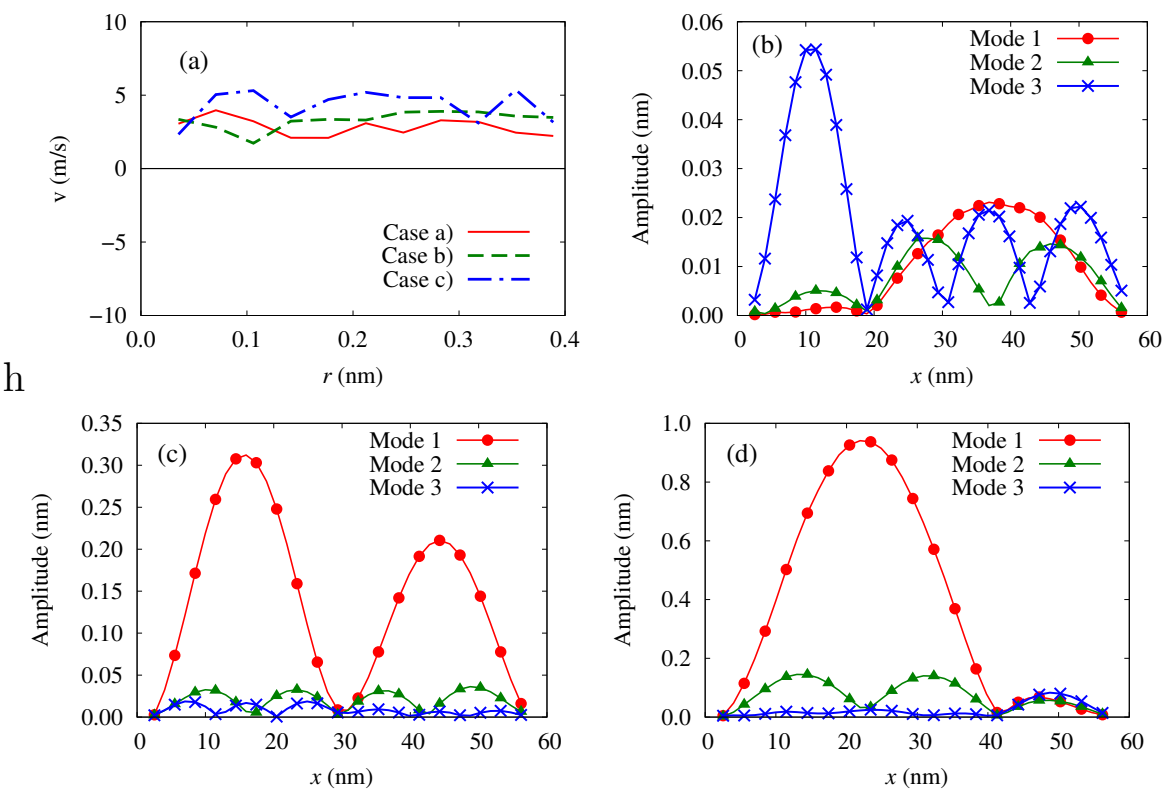

Figure 3: Velocity profiles and FFT analysis in a $60 \mathrm{~nm}$-long $(12,0) \mathrm{CNT}$ with fixed points as shown in Figure 1a, b and c. All CNTs had an imposed thermal gradient of $2 \mathrm{~K} / \mathrm{nm}$. (a) Radial distribution of axial velocity for the three different cases of Figure 1 (case a, b, c respectively) with mean velocities of $2.8 \mathrm{~m} / \mathrm{s}$ (red), $3.3 \mathrm{~m} / \mathrm{s}$ (green) and $4.5 \mathrm{~m} / \mathrm{s}$ (blue). (b) Amplitudes for vibrational modes 1-3 for CNT as shown in figure 1a (central fixed point at $1 / 3$ of the length). (c) Amplitudes for vibrational modes 1-3 for CNT as shown in Figure 1b (central fixed point at half the length), and (d) Amplitudes for vibrational modes 1-3 for CNT as shown in Figure 1c (central fixed point at 2/3 of the length). 
strongly affects the amplitudes of the first three vibrational modes under the same thermal gradient. Considering the reference case, i.e., the CNT with fixed end points and a fixed point in the middle, the corresponding amplitudes of the three vibrational modes are shown in Figure 3c. From this figure, we observe the same behavior as in Figure 2c, i.e., a direct impact of the thermal gradient on mode 1, with a greater amplitude in the high-temperature zone compared to the low-temperature zone. On the other hand, mode 2 is not affected by the imposed gradient, while mode 3 shows a slight increase in the amplitudes on the high-temperature zone relative to the low-temperature zone. In the case with the lowest flow rate measured, i.e. case (a), the amplitudes are presented in Figure 3b. This figure shows how the vibrational modes of the CNT are distorted with respect to the case with the central fixed point (Figure 3c); here the CNT acquires a greater freedom of movement in the low-temperature zone, leading to an increase of the amplitude of mode 1 in this zone. However, due to the particular direction of the flow, it follows that the third mode does drive the fluid. In fact, the effect of mode 3 on driving the fluid is the most significant when the CNT is restricted at $1 / 3$ of its length. Here, the frequency of mode 3 is $0.065 \mathrm{THz}$, which is more than four times the frequency of mode $1(0.015 \mathrm{Thz})$, leading to oscillations with a higher frequency and amplitude in the high-temperature zone as compared to the oscillations in the low-temperature zone. Finally, the amplitudes measured in the case with higher flow rate, i.e. case (c), are depicted in Figure $3 \mathrm{~d}$. This figure shows how the amplitude of mode 1 is strongly increased, more than twice compared to the peak amplitude of the corresponding mode in Figure 3c. Similar to Figure 3b, modes 2 and 3 are distorted by modifying the central fixed point, leading to a greater amplitude in the high-temperature zone for mode 2 , and in the low-temperature zone for mode 3 . The results for the three cases, indicate that the water flow is driven by an association between the frequencies and amplitudes of "activated" vibrational modes ${ }^{33}$ due to the particular fixed position of the point between the two ends. To confirm this driving mechanism, two additional cases of the $60 \mathrm{~nm}$-long $(12,0)$ CNT were simulated, with restrictions at $1 / 4$ and $1 / 5$ of the length, respectively. In 
both cases, a water flow with mean velocity of $c a .3 .5 \mathrm{~m} / \mathrm{s}$ was calculated. Moreover, in the high-temperature zone, a higher amplitude of mode 4 was computed for the case restricted at $1 / 4$ length, and similarly, a higher amplitude of mode 5 was computed in the case restricted at 1/5 length (see Supporting Information; Fig. S7 and Fig. S8). This indicates that the position of the pivotal fixed point with respect to the total length of the CNT determines the magnified harmonic vibrational mode driving the flow. Similar to the mechanism proposed by Qiu et al. ${ }^{28}$ we infer that an axial centrifugal force is propelling the water molecules. In our device, the magnitude of the centrifugal force is a consequence of the amplitudes and the frequency of a specific vibrational mode induced by the imposed thermal gradient and the particular position of the fixed middle point.

The feasibility of inducing continuous water flow in a CNT by imposing an external temperature gradient has not been widely investigated. In a recent study, Zhao and $\mathrm{Wu}^{18}$ showed that by keeping two reservoirs at different temperatures and connecting them with short aligned carbon nanotubes, net flow of water towards the low-temperature reservoir was observed. They reported significant higher flow rates for longer CNTs connecting the reservoirs, while keeping the end-to-end temperature difference constant (i.e. lower temperature gradient). This disagrees with the present results, since we compute lower flow rates for longer CNTs subjected to the same temperature difference (Supporting Information Fig. S8). This discrepancy is mainly related to the different treatment of the carbon atoms in the simulations, while Zhao and $\mathrm{Wu}^{18}$ imposed a harmonic restraining force to all the carbon atoms in the nanotube, in the present study, the nanotube vibrations are controlled without suppressing substantially the thermal oscillations of the nanotube. Additionally, the finite length of the CNT membrane in the study of Zhao and $\mathrm{Wu}^{18}$ leads to a higher energy barrier at the entrance, which is not taken into account in the present study.

To further explore this TBM, we evaluated the thermal pumping for CNTs with different diameters. Simulations of water in CNTs with diameters of $1.4 \mathrm{~nm}$ and $2.0 \mathrm{~nm}$, chirality vectors $(18,0)$ and $(26,0)$ respectively, were conducted. In both systems, a fixed thermal 
gradient of $3 \mathrm{~K} / \mathrm{nm}$ was imposed along the CNTs. Water velocity profiles along the axial direction for the $(18,0)$ and $(26,0)$ CNTs are shown in Figure 4. For both cases, the water flow displays a plug-like velocity profile with mean velocity of $c a .3 \mathrm{~m} / \mathrm{s}$. It is interesting to note that the exhibited independence of flow velocity on CNT diameter indicates that the proposed pump configuration (Figure 1b) may be, in principle, scalable to larger diameters. However, further investigation is required to confirm this hypothesis.

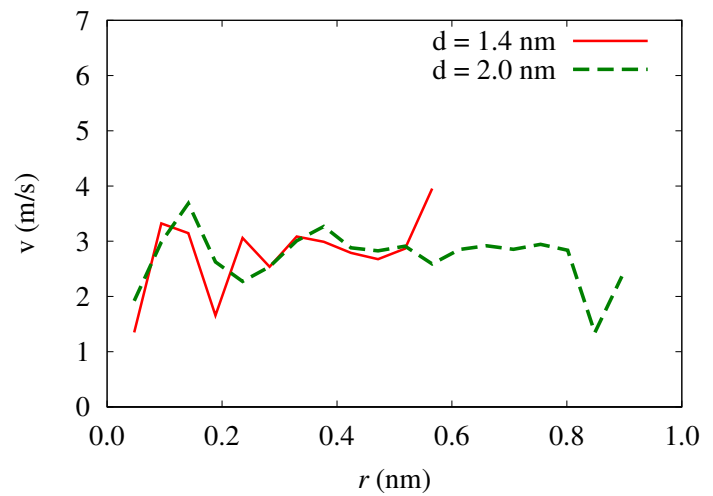

Figure 4: Radial distribution of axial velocity of water inside CNTs of $1.4 \mathrm{~nm}$ and $2.0 \mathrm{~nm}$ in diameter. The chiralities are $(18,0)$ and $(26,0)$, respectively. The applied thermal gradient is $3 \mathrm{~K} / \mathrm{nm}$ in both cases.

We believe that the present results provide valuable insight in the field of nanofluidic devices and also open the door to potential practical exploitation of thermal gradients for driving flow in nanoconduits. It should be noted that large thermal gradients are used in this study in order to increase the signal-to-noise ratio in our simulations, allowing us to extract measurable flow data without requiring prohibitively-long simulation times. In fact, for practical applications in nanofluidic devices wherein the typical distances are in the order of hundreds of nanometers, the relatively high temperature differences required to impose such large gradients in the CNT conduits, could give rise to some concern about the boiling temperature of water. Nevertheless, recent studies have reported that water phase transitions under nanoconfinement may deviate from classical behavior. ${ }^{34,35}$ Specifically, the temperature for liquid-vapor transition of water confined in CNTs with similar diameters used in the present study is substantially raised above $100^{\circ} \mathrm{C}$ due to nanoconfinement. Therefore, 
we infer that if a net water flow can be produced imposing a gradient of $0.5 \mathrm{~K} / \mathrm{nm}$ (see Supporting information Figure S15) and assuming the boiling temperature is significantly higher inside the CNT then, the system proposed here could pump liquid water through CNTs with lengths of ca. $500 \mathrm{~nm}$. Moreover, nanofabrication techniques currently allow the fabrication of ultrathin membranes connecting two reservoirs separated only by $100 \mathrm{~nm} .{ }^{36}$ This type of ultrathin membranes can be used in combination with vertically-oriented $\mathrm{CNTs}^{37}$ for molecular sieving applications or for separation of analytes immersed in water solutions, wherein the flow is driven by thermal gradients, as in the present work.

\section{Conclusion}

Using MD simulations, we have investigated the capability of CNTs subjected to a thermal gradient, to sustain continuous and fast water transport in their interior. This study provides the basis for developing a thermal pump based on single-wall carbon nanotubes. The device is able to pump continuous flows with average velocity up to $5 \mathrm{~m} / \mathrm{s}$. The mechanisms driving the fluid flow are thermally-induced asymmetric oscillations along the CNT, which propel the fluid in a constant, whip-like motion. Flow rate control is achieved by the direction and magnitude of the imposed temperature gradient and by modifying the position of the pivotal fixed point along the CNT. The interplay between relative positions of the fixed points and the applied thermal gradient produce greater amplitudes in the high-temperature zone compared to the low-temperature zone for specific vibrational modes. We envision that CNTs with thermal gradients could assist the design of nano-chips that require fast water transport between their components.

\section{Methods}

To study the rectified flow of water driven by thermal gradients inside the CNT, we perform a series of all-atom MD simulations. The simulations are performed using the MD package 
FASTTUBE. ${ }^{38}$ The equations of motion are integrated in time using the leapfrog scheme with a time step of $2 \mathrm{fs}$. All simulations are conducted in an orthorhombic box with periodic boundary conditions in the axial direction of the CNT and free space conditions in the radial direction. The carbon-carbon intramolecular interactions of the CNT are described by a Morse bond, a harmonic cosine of the bending angle, and a torsion potential. ${ }^{16,38,39}$ Water is modeled by the rigid SPC/E model ${ }^{40}$ and the water-CNT interactions are described by a 12-6 LJ potential calibrated for a $81^{\circ}$ contact angle. ${ }^{16,41}$ The van der Waals and Coulomb interactions are truncated at $1 \mathrm{~nm}$, while the Coulomb potential is smoothed to ensure energy conservation. ${ }^{16,38}$ The MD package and the force fields have been extensively validated in previous works. ${ }^{15-17,38,39,41,42}$ For details of the potentials, we refer the reader to Zambrano et al. ${ }^{16}$

We first equilibrate the system at $300 \mathrm{~K}$ in the NVT ensemble for $0.5 \mathrm{~ns}$. Then, using nonequilibrium molecular dynamics (NEMD) simulations, we impose a thermal gradient along the CNT axis. The thermal gradient is imposed by applying two different temperatures at the respective ends of the CNT, as depicted in Figure 1. Specifically, the carbon atoms in each heated zone are coupled to Berendsen thermostats. ${ }^{43}$ It is important to note that the water molecules are not connected to the thermostat in the NEMD simulations. Previous studies have demonstrated that the Berendsen thermostat is suitable to impose proper nonequilibrium conditions, ${ }^{44,45}$ and is optimal for mechanical responses at relatively constant temperature during CNT compression. ${ }^{46}$ In order to remove spurious effects of the thermostat, the mean velocity of the heated carbon atoms is subtracted, and subsequently added, when the thermostat is applied. We conduct the NEMD simulations during 100 ns to reduce thermal noise, and ensure a steady water flow rate. From the atomic trajectories, the CNT vibrations are analyzed by a FFT algorithm, measuring the amplitudes and frequencies of the different vibrational modes. This FFT method was previously proposed by Pine et al. ${ }^{47}$ Further details on the simulations and FFT analysis are presented in the Supporting Information. 


\section{Acknowledgement}

This research was funded by CONICYT (Chile) under FONDECYT Project No. 11130559 and under CONICYT scholarship No. 21140427. We acknowledge partial financial support from the University of Concepcion under VRID Project No. 21496651. The authors received computational support from the Department of Physics at the Technical University of Denmark.

\section{Supporting Information Available}

Simulation protocol, summary of all simulated cases, temperature profile, vibrational (FFT) analysis, water flow vs CNT length, efficiency computation, simulations without fixing points and water flow as a function of the central fixed point position.

\section{References}

1. Abgrall, P.; Gue, A. Lab-On-Chip Technologies: Making a Microfluidic Network and Coupling it Into a Complete Microsystem - A Review. J. Micromech. Microeng. 2007, 17, R15.

2. Abgrall, P.; Nguyen, N. T. Nanofluidic Devices and Their Applications. Anal. Chem. 2008, 80, 2326-2341.

3. Majumder, M.; Chopra, N.; Andrews, R.; Hinds, B. J. Nanoscale Hydrodynamics: Enhanced Flow in Carbon Nanotubes. Nature 2005, 438, 44-44.

4. Holt, J. K.; Park, H. G.; Wang, Y.; Stadermann, M.; Artyukhin, A. B.; Grigoropoulos, C. P.; Noy, A.; Bakajin, O. Fast Mass Transport Through Sub-2-Nanometer Carbon Nanotubes. Science 2006, 312, 1034-1037. 
5. Walther, J. H.; Ritos, K.; Cruz-Chu, E. R.; Megaridis, C. M.; Koumoutsakos, P. Barriers to Superfast Water Transport in Carbon Nanotube Membranes. Nano Lett. 2013, 13, $1910-1914$.

6. Popov, V. N. Carbon Nanotubes: Properties and Application. Mat. Sci. Eng., R 2004, $43,61-102$.

7. Kral, P.; Wang, B. Material Drag Phenomena in Nanotubes. Chem. Rev. 2013, 113, $3372-3390$.

8. Thomas, J. A.; McGaughey, A. J. H. Water Flow in Carbon Nanotubes: Transition to Subcontinuum Transport. Phys. Rev. Lett. 2009, 102, 184502.

9. Joseph, S.; Aluru, N. Pumping of Confined Water in Carbon Nanotubes by RotationTranslation Coupling. Phys. Rev. Lett. 2008, 101, 064502.

10. Wang, Y.; Zhao, Y.; Huang, J. Giant Pumping of Single-File Water Molecules in a Carbon Nanotube. J. Phys. Chem. B 2011, 115, 13275-13279.

11. Azamat, J.; Sardroodi, J.; Rastkar, A. Water Desalination Through Armchair Carbon Nanotubes: A Molecular Dynamics Study. RSC Adv. 2014, 4, 63712-63718.

12. Joseph, S.; Aluru, N. Why Are Carbon Nanotubes Fast Transporters of Water? Nano Lett. 2008, 8, 452-458.

13. Berber, S.; Kwon, Y.-K.; Tománek, D. Unusually High Thermal Conductivity of Carbon Nanotubes. Phys. Rev. Lett. 2000, 84, 4613.

14. Barreiro, A.; Rurali, R.; Hernandez, E. R.; Moser, J.; Pichler, T.; Forro, L.; Bachtold, A. Subnanometer Motion of Cargoes Driven by Thermal Gradients Along Carbon Nanotubes. Science 2008, 320, 775-778. 
15. Schoen, P. A.; Walther, J. H.; Arcidiacono, S.; Poulikakos, D.; Koumoutsakos, P. Nanoparticle Traffic on Helical Tracks: Thermophoretic Mass Transport Through Carbon Nanotubes. Nano Lett. 2006, 6, 1910-1917.

16. Zambrano, H. A.; Walther, J. H.; Koumoutsakos, P.; Sbalzarini, I. F. Thermophoretic Motion of Water Nanodroplets Confined Inside Carbon Nanotubes. Nano Lett. 2009, 9, 66-71.

17. Zambrano, H. A.; Walther, J. H.; Jaffe, R. L. Thermally Driven Molecular Linear Motors: A Molecular Dynamics Study. J. Chem. Phys. 2009, 131, 241104.

18. Zhao, K.; Wu, H. Fast Water Thermo-Pumping Flow Across Nanotube Membranes for Desalination. Nano Lett. 2015, 15, 3664-3668.

19. Hou, Q.-W.; Cao, B.-Y.; Guo, Z.-Y. Thermal Gradient Induced Actuation in DoubleWalled Carbon Nanotubes. Nanotechnology 2009, 20, 495503.

20. Prasad, M. V.; Bhattacharya, B. Phonon Scattering Dynamics of Thermophoretic Motion in Carbon Nanotube Oscillators. Nano Lett. 2016, 16, 2174-2180.

21. Chen, J.; Gao, Y.; Wang, C.; Zhang, R.; Zhao, H.; Fang, H. Impeded Mass Transportation Due to Defects in Thermally Driven Nanotube Nanomotor. J. Phys. Chem. C 2015, 119, 17362-17368.

22. Rurali, R.; Hernandez, E. Thermally Induced Directed Motion of Fullerene Clusters Encapsulated in Carbon Nanotubes. Chem. Phys. Lett. 2010, 497, 62-65.

23. Guo, Z.; Chang, T.; Guo, X.; Gao, H. Mechanics of Thermophoretic and Thermally Induced Edge Forces in Carbon Nanotube Nanodevices. J. Mech. Phys. Solids 2012, 60, $1676-1687$.

24. Santamaría-Holek, I.; Reguera, D.; Rubi, J. Carbon-Nanotube-Based Motor Driven by a Thermal Gradient. J. Phys. Chem. C 2013, 117, 3109-3113. 
25. Wei, N.; Wang, H.-Q.; Zheng, J.-C. Nanoparticle Manipulation by Thermal Gradient. Nanoscale Res. Lett. 2012, 7, 1-9.

26. Erbas-Cakmak, S.; Leigh, D. A.; McTernan, C. T.; Nussbaumer, A. L. Artificial Molecular Machines. Chem. Rev. 2015, 115, 10081-10206.

27. Insepov, Z.; Wolf, D.; Hassanein, A. Nanopumping Using Carbon Nanotubes. Nano Lett. 2006, 6, 1893-1895.

28. Qiu, H.; Shen, R.; Guo, W. Vibrating Carbon Nanotubes as Water Pumps. Nano Res. 2011, 4, 284-289.

29. Tu, Z. Efficiency at Maximum Power of Feynman's Ratchet as a Heat Engine. J. Phys. A: Math. Theor. 2008, 41, 312003.

30. Parrondo, J. M.; Blanco, J.; Cao, F.; Brito, R. Efficiency of Brownian Motors. Europhys. Lett. 1998, 43, 248.

31. Hänggi, P.; Marchesoni, F. Artificial Brownian Motors: Controlling Transport on the Nanoscale. Rev. Mod. Phys. 2009, 81, 387.

32. Becton, M.; Wang, X. Thermal Gradients on Graphene to Drive Nanoflake Motion. J. Chem. Theory Comput. 2014, 10, 722-730.

33. Bocquet, L.; Netz, R. R. Nanofluidics: Phonon modes for Faster Flow. Nature Nanotechnol. 2015, 10, 657-658.

34. Chaban, V. V.; Prezhdo, O. V. Water Boiling Inside Carbon Nanotubes: Toward Efficient Drug Release. ACS Nano 2011, 5, 5647-5655.

35. Agrawal, K. V.; Shimizu, S.; Drahushuk, L. W.; Kilcoyne, D.; Strano, M. S. Observation of Extreme Phase Transition Temperatures of Water Confined Inside Isolated Carbon Nanotubes. Nature Nanotechnol. 2017, 12, 267-273. 
36. Lin, X.; Yang, Q.; Ding, L.; Su, B. Ultrathin Silica Membranes with Highly Ordered and Perpendicular Nanochannels for Precise and Fast Molecular Separation. ACS Nano 2015, 9, 11266-11277.

37. Majumder, M.; Chopra, N.; Hinds, B. J. Mass Transport Through Carbon Nanotube Membranes in Three Different Regimes: Ionic Diffusion and Gas and Liquid Flow. ACS Nano 2011, 5, 3867-3877.

38. Walther, J. H.; Jaffe, R.; Halicioglu, T.; Koumoutsakos, P. Carbon Nanotubes in Water: Structural Characteristics and Energetics. J. Phys. Chem. B 2001, 105, 9980-9987.

39. Schoen, P. A.; Walther, J. H.; Poulikakos, D.; Koumoutsakos, P. Phonon Assisted Thermophoretic Motion of Gold Nanoparticles Inside Carbon Nanotubes. Appl. Phys. Lett. 2007, 90, 253116.

40. Berendsen, H. J. C.; Grigera, J. R.; Straatsma, T. P. The Missing Term in Effective Pair Potentials. J. Phys. Chem. 1987, 91, 6269-6271.

41. Werder, T.; Walther, J. H.; Jaffe, R. L.; Halicioglu, T.; Koumoutsakos, P. On the WaterGraphite Interaction for Use in MD Simulations of Graphite and Carbon Nanotubes. J. Phys. Chem. B 2003, 107, 1345-1352.

42. Werder, T.; Walther, J. H.; Jaffe, R.; Halicioglu, T.; Noca, F.; Koumoutsakos, P. Molecular Dynamics Simulations of Contact Angles of Water Droplets in Carbon Nanotubes. Nano Lett. 2001, 1, 697-702.

43. Berendsen, H. J. C.; Postma, J. P. M.; van Gunsteren, W. F.; DiNola, A.; Haak, J. R. Molecular Dynamics with Coupling to an External Bath. J. Chem. Phys. 1984, 81, $3684-3684$.

44. Berendsen, H. J. Simulating the Physical World: Hierarchical Modeling from Quantum Mechanics to Fluid Dynamics; Cambridge University Press, 2007; pp 195, 203. 
45. Van Der Spoel, D.; Lindahl, E.; Hess, B.; Groenhof, G.; Mark, A. E.; Berendsen, H. J. GROMACS: Fast, Flexible, and Free. J. Comput. Chem. 2005, 26, 1701-1718.

46. Heo, S.; Sinnott, S. B. Investigation of the Influence of Thermostat Configurations on the Mechanical Properties of Carbon Nanotubes in Molecular Dynamics Simulations. J. Nanosci. Nanotechnol. 2007, 7, 1518-1524.

47. Pine, P.; Yaish, Y. E.; Adler, J. Simulation and Vibrational Analysis of Thermal Oscillations of Single-Walled Carbon Nanotubes. Phys. Rev. B 2011, 83, 155410. 
372 Graphical TOC Entry

373

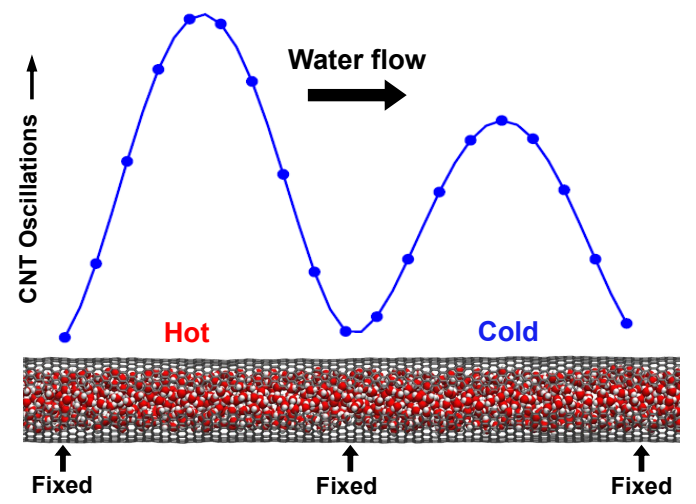

